\title{
Delta-operator-based adaptive control of a piston pump
}

\author{
FUZHENG HU and KEVIN EDGE \\ School of Mechanical Engineering \\ University of Bath \\ Bath, BA2 7AY \\ England
}

\begin{abstract}
This paper describes the application of a delta-operator-based model reference adaptive control scheme to the control of the delivery pressure of a variable capacity swash plate piston pump. It has been found from the simulation results that good model following performance can be achieved. The nonminimum phase problem due to fast sampling can be solved through the use of a deltaoperator-based adaptive control scheme, instead of the usual back shift operator. Disturbance rejection properties are limited by signal saturation in the pump displacement servo system.
\end{abstract}

\section{KEY WORDS}

Delta operator, Adaptive control, Piston pump

\section{NOTATION}

$\mathrm{A}(\delta) \quad$ Characteristic polynomial of the discretized model of plant

$A_{m}(\delta)$ Characteristic polynomial of the reference model

$\mathrm{B}(\delta)$ numerator polynomial of the discretized model of plant

$\mathrm{B}_{\mathrm{m}}(\delta)$ numerator polynomial of the reference model

$\mathrm{B}_{\mathrm{R}}(\delta)$ Suitable approximation of $\mathrm{B}(\delta)$

$\mathrm{B}_{\Delta}(\delta)=\mathrm{B}(\delta)-\mathrm{B}_{\mathrm{R}}(\delta)$

$\mathrm{e}(\mathrm{k}) \quad=\mathrm{y}_{\mathrm{m}}(\mathrm{k})-\mathrm{y}_{\mathrm{p}}(\mathrm{k})$, tracking error

$\mathrm{E}(\delta) \quad=\mathrm{F}(\delta)-\mathrm{A}(\delta)$, polynomial

$\mathrm{F}(\delta) \quad$ Characteristic polynomial of state filter h Sampling period

$\mathrm{k}$ discrete time interval

L Number of the unstable zeros for the discretized plant

$m \quad$ Number of zeros of the continuous time plant

n Order of the plant to be controlled

$\mathrm{P}(\delta) \quad$ Polynomial used for controller design

$\mathrm{Q}(\delta) \quad$ Polynomial used for controller design

$\mathrm{r}(\mathrm{k}) \quad$ Reference input

$\mathrm{u}(\mathrm{k}) \quad$ Control input

$y_{m}(k) \quad$ Output of the reference model

$y_{p}(k) \quad$ Output of the plant to be controlled

$\mathrm{z} \quad$ Forward shift operator

$\delta=(\mathrm{z}-1) / \mathrm{h}$, delta operator 


\section{INTRODUCTION}

Because both classical and optimal control schemes are unable to accommodate variations or uncertainties in circuit parameters, the application of adaptive control schemes to hydraulic systems has been widely studied for the improvement of hydraulic system dynamics eg [1-3]. However, because electro-hydraulic systems usually have a high relative order, their z-transfer functions usually have unstable zeros unless an unreasonably large sampling period is used [4]. With rapid sampling, the back-shiftbased model reference control schemes based on zero cancellation can not be used because the control input will go boundless. Also, a large sampling period usually results in poor, or even unacceptable control performance and disturbance rejection properties. With the aim of solving the above-mentioned problems on adaptive control for discrete nonminimum phase systems due to certain small sampling periods, the idea of using a delta operator, instead of the usual back shift operator has been proposed by Goodwin et al.[5], Janecki [6], and Suzuki and Tanaka [7]. In this paper, the application of a delta-operator-based model reference adaptive scheme to the control of the delivery pressure of a variable capacity swash plate piston pump is studied on the basis of computer simulations.

\section{ADAPTIVE CONTROLLER}

Two adaptive controllers using the delta operator have been presented in references [5], and [6]. In this paper, the controller proposed in [5] will be slightly modified and applied to the control of a piston pump.

It is assumed that a single-input, singleoutput, continuous-time plant with $\mathrm{n}$ poles and $m$ zeros and without external disturbances is sampled at the sampling period $h$, and the digital control input acts on the continuous time plant via a zero-order hold. Thus the deltaoperator-based discretized representation for the plant can be obtained as:

$$
\mathrm{A}(\delta) \mathrm{y}_{\mathrm{p}}(\mathrm{k})=\mathrm{B}(\delta) \mathrm{u}(\mathrm{k})
$$

where polynomials $A(\delta)$ and $B(\delta)$ of degree $n$ and $n-1$ can be expressed as:

$$
\begin{aligned}
& \mathrm{A}(\delta)=\delta^{\mathrm{n}}+\mathrm{A}_{\mathrm{n}-1} \delta^{\mathrm{n}-1}+\ldots+\mathrm{A}_{1} \delta+\mathrm{A}_{0} \\
& \mathrm{~B}(\delta)=\mathrm{B}_{\mathrm{n}-1} \delta^{\mathrm{n}-1}+\ldots+\mathrm{B}_{\mathrm{m}} \delta^{\mathrm{m}}+\ldots+\mathrm{B}_{0}
\end{aligned}
$$

The task of a model reference adaptive controller is to control the plant defined by (1) so that the output of the plant closely tracks the output of the reference model given by:

$$
A_{m}(\delta) y_{m}(k)=B_{m}(\delta) r(k)
$$

where $A_{m}(\delta)$ and $B_{m}(\delta)$ are polynomials and the selection of their orders will be discussed later.

The basic structure of adaptive controller based on zero and pole cancellation can be obtained by the analogy to those for general continuous time plants as follows [5, 8]:

$$
\mathrm{P}(\delta) \mathrm{B}(\delta) \mathrm{u}(\mathrm{k})=\mathrm{B}_{\mathrm{m}}(\delta) \mathrm{r}(\mathrm{k})-\mathrm{Q}(\delta) \mathrm{y}_{\mathrm{p}}(\mathrm{k})
$$

where polynomials $\mathrm{P}(\delta)$ and $\mathrm{Q}(\delta)$ satisfy the polynomial equation:

$$
A_{m}(\delta)=P(\delta) A(\delta)+Q(\delta)
$$

In equation (6), $\mathrm{Q}(\delta)$ has a degree of $\mathrm{n}-1$. The degree of $\mathrm{P}(\delta)$ depends on that of $\mathrm{A}_{\mathrm{m}}(\delta)$.

Unfortunately, when $\mathrm{B}(\delta)$ has unstable zeros at some small sampling period, the controller structure defined by (5) and (6) cannot be used because in this case $\mathrm{u}(\mathrm{k})$ will be unstable. Therefore, a suitable approximation for $\mathrm{B}(\delta)$ is needed in order to be able to use the above controller structure.

$\mathrm{B}(\delta)$ can be rewritten as the summation of two terms as follows: 


$$
\mathrm{B}(\delta)=\mathrm{B}_{\Delta}(\delta)+\mathrm{B}_{\mathrm{R}}(\delta)
$$

where $\mathrm{B}_{\mathrm{R}}(\delta)$ and $\mathrm{B}_{\Delta}(\delta)$ are polynomials defined as follows:

$$
\begin{aligned}
& \mathrm{B}_{\Delta}(\delta)=\mathrm{B}_{\mathrm{n}-1} \delta^{\mathrm{n}-1}+\ldots \ldots+\mathrm{B}_{\mathrm{m}+1} \delta^{\mathrm{m}+1} \\
& \mathrm{~B}_{\mathrm{R}}(\delta)=\mathrm{B}_{\mathrm{m}} \delta^{\mathrm{m}}+\ldots \ldots+\mathrm{B}_{1} \delta+\mathrm{B}_{0}
\end{aligned}
$$

It has been proven that the zeros of $B_{R}(\delta)$ will approach those of the continuous time plant and $\mathrm{B}_{\Delta}(\delta)$ will approach zero as the sampling period $h$ tends to zero $[5,6]$. Therefore, a reasonable substitute for $\mathrm{B}(\delta)$ is obviously $\mathrm{B}_{\mathrm{R}}(\delta)$, which is proposed in [5]. However, in practical applications, the sampling period $h$ cannot approach zero. In some cases, lower order terms of $\mathrm{B}_{\Delta}(\delta)$ are quite large and completely neglecting $\mathrm{B}_{\Delta}(\delta)$ may result in a large error in the discretized model so that the stability and performance of the adaptive scheme deteriorates. In order to reduce the error in the model, it is desirable that $B_{\Delta}(\delta)$ to be neglected contains as few terms as possible. On the other hand, it is known that if $B_{\Delta}(\delta)$ includes too few terms, $B_{R}(\delta)$ will have unstable zeros. A reasonable compromise has to be considered here.

It is known from [4] that when the sampling period is sufficiently small the polynomial $\mathrm{B}(\delta)$ in (1) has $\mathrm{m}$ dominant stable zeros, which correspond to those of the continuous time plant, and $n-m-1$ parasitic zeros, which arise from discretization. Also, it can easily be inferred from reference [4] that all the unstable parasitic zeros of $\mathrm{B}(\delta)$ are negatively real and are located to the left-hand side of the stable area, which is a circle of radius $1 / \mathrm{h}$ and centred on point $(-1 / \mathrm{h}, 0)$ in the delta plane. In most cases the limit of the number of unstable parasitic zeros, $\mathrm{L}<\mathrm{n}-\mathrm{m}-1$, is known when the relative order, $n-m$, of the continuous-time plant is known. Consequently, an improved stable $B_{R}(\delta)$ can be obtained by simply truncating $\mathrm{L}$ leading higher order terms of $\mathrm{B}(\delta)$. In this way the zeros of $\mathrm{B}_{\mathrm{R}}(\delta)$ will be located in the vicinity of all stable zeros of
$\mathrm{B}(\delta)$, including stable parasitic zeros, and will approach these stable zeros as the sampling period tends to zero. Thus, $\mathrm{B}_{\mathrm{R}}(\delta)$ can be expressed as:

$$
\mathrm{B}_{\mathrm{R}}(\delta)=\mathrm{B}_{\mathrm{n}-\mathrm{L}-1} \delta^{\mathrm{n}-\mathrm{L}-1}+\cdots+\mathrm{B}_{\mathrm{m}} \delta^{\mathrm{m}}+\ldots+\mathrm{B}_{0}
$$

Correspondingly, $\mathrm{B}_{\Delta}(\delta)$ can be rewritten as:

$$
\mathrm{B}_{\Delta}(\delta)=\mathrm{B}_{\mathrm{n}-1} \delta^{\mathrm{n}-1}+\ldots+\mathrm{B}_{\mathrm{n}-\mathrm{L}} \delta^{\mathrm{n}-\mathrm{L}}
$$

The control law given by equation (5) becomes:

$$
\mathrm{P}(\delta) \mathrm{B}_{\mathrm{R}}(\delta) \mathrm{u}(\mathrm{k})=\mathrm{B}_{\mathrm{m}}(\delta) \mathrm{r}(\mathrm{k})-\mathrm{Q}(\delta) \mathrm{y}_{\mathrm{p}}(\mathrm{k})
$$

In order to reduce the order of the controller defined by (12), the degree of $B_{m}(\delta)$ should be selected to be less than or equal to that of $Q(\delta)$ $\mathrm{n}-1$. In this case, $\mathrm{P}(\delta)$ must have a degree larger than or equal to $\mathrm{L}$ for implementation of the controller (12). Therefore, it is known from equation (6) that the degree of $A_{m}(\delta)$ should be larger than or equal to $\mathrm{n}+\mathrm{L}$.

In order to implement the indirect model reference adaptive controller given by (12) and $(6), \mathrm{B}_{\mathrm{R}}(\delta)$ and $\mathrm{A}(\delta)$ have to be identified on line.

For the monic polynomial $A(\delta)$ defined by equation (2), there exists an arbitrary stable monic polynomial of degree $n, F(\delta)$ and a polynomial of degree $n-1, E(\delta)$ such that

$$
\mathrm{F}(\delta)=\mathrm{A}(\delta)+\mathrm{E}(\delta)
$$

By multiplying equation (13) by $\mathrm{y}_{\mathrm{p}}(\mathrm{k})$, and then using equation (1), it follows that

$$
\mathrm{y}_{\mathrm{p}}(\mathrm{k})=\mathrm{B}(\delta) \mathrm{u}_{\mathrm{f}}(\mathrm{k})+\mathrm{E}(\delta) \mathrm{y}_{\mathrm{pf}}(\mathrm{k})
$$

where $\mathrm{u}_{\mathrm{f}}(\mathrm{k})$ and $\mathrm{y}_{\mathrm{pf}}(\mathrm{k})$ are respectively the filtered values of $u(k)$ and $y_{p}(k)$ via $F(\delta)$, and can be expressed as:

$$
\begin{aligned}
& \mathrm{u}_{\mathrm{f}}(\mathrm{k})=\mathrm{u}(\mathrm{k}) / \mathrm{F}(\delta) \\
& \mathrm{y}_{\mathrm{pf}}(\mathrm{k})=\mathrm{y}_{\mathrm{p}}(\mathrm{k}) / \mathrm{F}(\delta)
\end{aligned}
$$


Using the equation (14), the generalized adaptive laws presented in [7] can be used to adjust the coefficients of $\mathrm{B}(\delta)$ and $\mathrm{E}(\delta)$. The estimate of $\mathrm{A}(\delta)$ can be obtained from equation (13) because $F(\delta)$ is a given polynomial.

As the plant parameters are identified online, the control input can be generated by (12) and (6).

However, the stability of $\mathrm{u}(\mathrm{k})$ still needs further consideration.

If the parameter adjustment is convergent, using (1) and (6), equation (12) can be rewritten in the form of a transfer function as:

$$
u(k)=\frac{A(\delta) B_{m}(\delta) r(k)}{A_{m}(\delta) B_{R}(\delta)+Q(\delta) B_{\Delta}(\delta)}
$$

Although $\mathrm{B}(\delta)$ is not a common factor of the characteristic polynomial in (17), the stability of $\mathrm{u}(\mathrm{k})$ is still dependent on $\mathrm{B}_{\Delta}(\delta)$. Obviously, in the case where $\mathrm{B}_{\Delta}(\delta)$ approaches zero as the sampling period $\mathrm{h}$ approaches zero, $\mathrm{u}(\mathrm{k})$ will be is stable because both $A_{m}(\delta)$ and $B_{R}(\delta)$ are stable polynomials in (17). Therefore, there must exist $\mathrm{h}_{0}>0$ such that the controlling input defined by (12) is stable for all $h<h_{0}$. In practice, because $h$ cannot really approach zero, the influence of $\mathrm{B}_{\Delta}(\delta)$ on the stability of $\mathrm{u}(\mathrm{k})$ cannot be neglected when $h$ is not very small. In order to guarantee the stability of the deltaoperator-based adaptive controller, the sampling period should be taken to be as small as possible. This is in fact a trade-off between stability and realizability. It is evident that the substitution of $\mathrm{B}_{\mathrm{R}}(\delta)$ defined by (9) with one defined by (10) can improve the stability of $\mathrm{u}(\mathrm{k})$ because the effects of $\mathrm{B}_{\Delta}(\delta)$ have been minimized.

After adaptation is complete, the tracking error can be expressed as:

$$
A_{m}(\delta) e(k)=-P(\delta) B_{\Delta}(\delta) u(k)
$$

It is clear from equation (18) that $\mathrm{e}(\mathrm{k})$ will be bounded if $\mathrm{u}(\mathrm{k})$ is stable and bounded because $\mathrm{A}_{\mathrm{m}}(\delta)$ is a stable polynomial. It also follows from equation $(18)$ that $\mathrm{e}(\mathrm{k})$ will tend to zero because $\mathrm{B}_{\Delta}(\delta)$ approaches zero as the sampling period $\mathrm{h}$ tends to zero.

\section{PUMP CONTROL}

The adaptive pressure control of a piston pump with the adaptive controller in the back shift operator is presented in [1]. In reference [1], a sampling period of 10 milliseconds has to be used to guarantee a minimum phase discretized plant model. Therefore, the bandwidth of the whole closed loop system has been limited to $20 \mathrm{rad} / \mathrm{s}$. Simulations indicate that when the sampling period is smaller than 10 milliseconds the discretized model of the plant under study has unstable zeros. With the aim of increasing the speed of response of the plant and improving disturbance rejection properties through the use of shorter sampling periods, the above indirect adaptive controller in the $\delta$ operator has been used for a simulation study of the delivery pressure control of a piston pump.

The plant under study is shown in FIGURE 1.

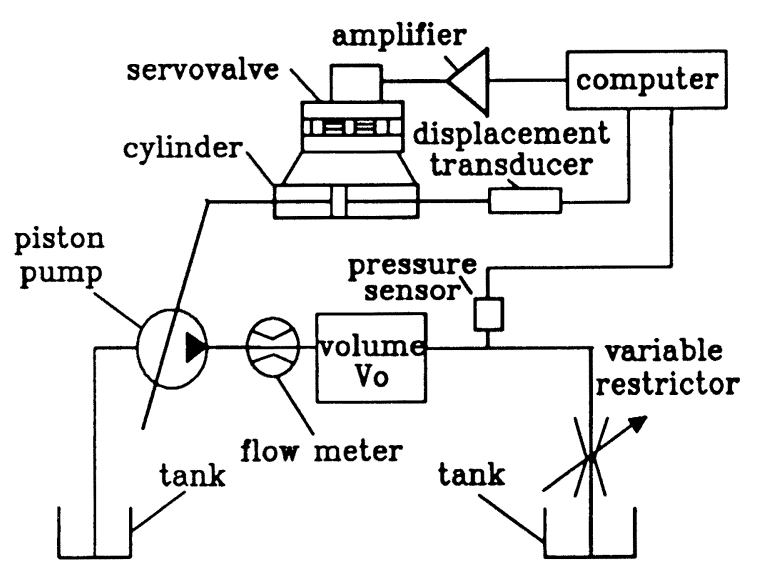

FIGURE 1. Plant under study

A servo-valve controlled cylinder is employed to position the pump swash plate and feedback is obtained from a pressure transducer mounted in the pump delivery line. A cylinder displacement transducer may be employed to provide an additional feedback signal, although 
it is not used in the scheme described below. The computer is programmed to implement the adaptive control scheme and the control signal drives the servo-valve through an amplifier in the usual manner. For the study reported here, it is assumed that the pump load consists a lumped volume and a variable restrictor valve. The objective of the controller is maintain the required pressure even in the presence of changes in the load valve setting.

The dynamic mathematical model of a piston pump control system is given in detail in reference [1]. The plant is simplified to a sixth order system with some nonlinearities. Here second order plant dynamics will be assumed, and the neglected dynamics will be treated as the unmodelled dynamics. The reference model was critically damped with a natural frequency of $100 \mathrm{rad} / \mathrm{s}$; the sampling period 0.001 second.

It is clear from FIGURE 2 and FIGURE 3 that the plant has very good output behaviour for both step and sinusoidal demand signals, as illustrated by the thin solid lines. The control inputs are stable for both cases, as shown by the dashed lines, and the tracking error is very small, as indicated by the dotted lines. Because a smaller sampling period is used the speed of response of the plant has been increased over that presented in [1] through the selection of a faster reference model.

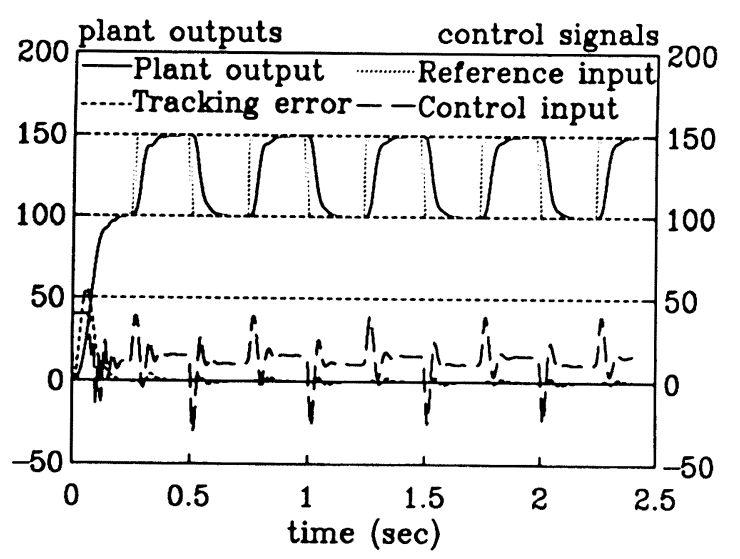

FIGURE 2. Step response of the plant under adaptive control

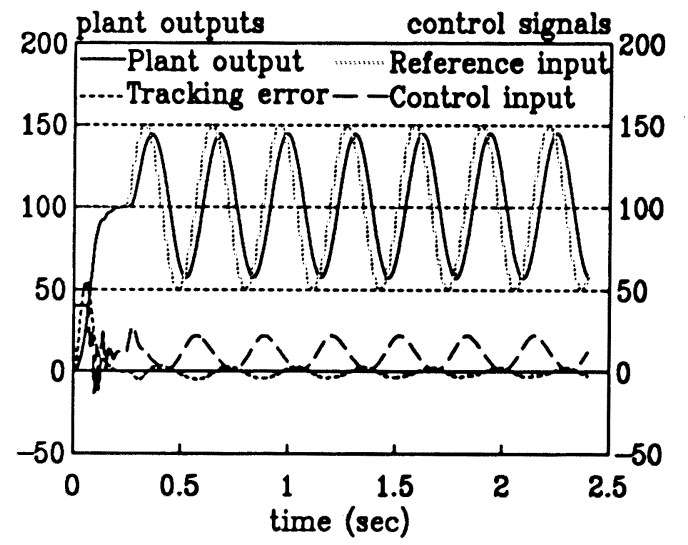

FIGURE 3. Sinusoidal response of the plant under adaptive control

FIGURE 4 and FIGURE 5 shows the disturbance rejection properties in the case of a $30 \%$ variation in the restrictor opening. It can be seen from FIGURE 4 and FIGURE 5 that the control inputs are stable and bounded for both step and sinusoidal variations in the restrictor opening. The tracking errors for both cases are reasonable and acceptable though a large overshoot (about 15\%) can be observed when the restrictor opening changes suddenly.

Although the sampling period has been decreased to one-tenth of that used in [1], the disturbance rejection properties are not significantly improved. The step variation in the restrictor opening leads to a rapid disturbance response and a sudden change in plant parameters. Improvement in the disturbance rejection properties is dependent on the speed of response of the whole closedloop system and the speed of parameter adaptation. Simulations indicate that the speed of the whole system plays a dominant role in rejecting the step disturbances in the restrictor opening. Although the speed of response can be theoretically increased by selecting a faster reference model, the improvement is limited because of valve signal saturation. A small sampling period only provides a possibility of using a faster reference model. Here, because 
of limitation of the servo actuator dynamics, a much faster reference model cannot be selected to reduce the overshoot caused by the step disturbance.

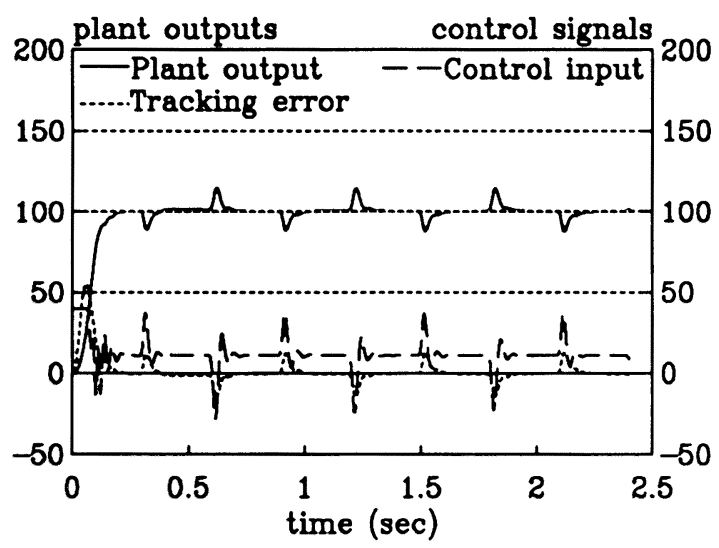

FIGURE 4. Plant performance under 30\% step variation in restrictor opening

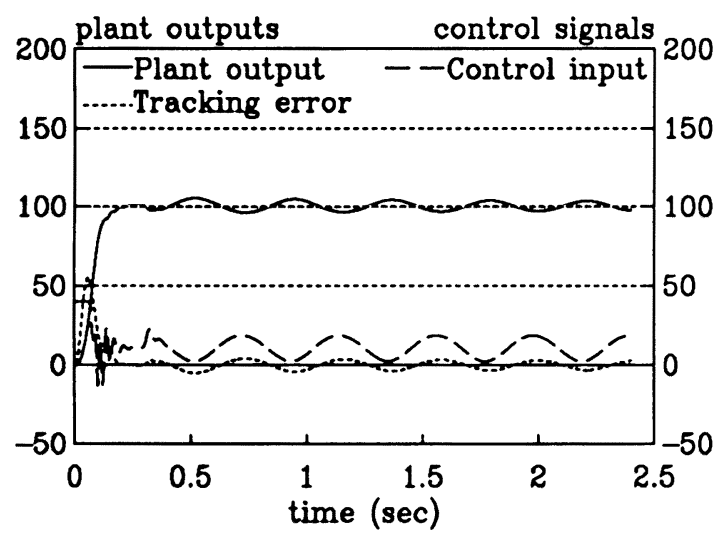

FIGURE 5. Plant performance under $30 \%$ sinusoidal variation in restrictor opening

\section{CONCLUSIONS}

From the preliminary simulation results given in the previous section, it can be concluded that

(1) Through the use of the delta operator, a much smaller sampling period can be used, and consequently the bandwidth of the whole closed-loop system can be extended.

(2) Adaptive controllers in the delta operator have very good performance, even in the case where unmodelled dynamics exist.

(3) The outstanding disadvantage is that adaptive schemes in the delta operator substantially increase calculations in comparison with those in the back-shift operator. This leads to difficulties in the realization of adaptive schemes, especially when the plant to be controlled is a high order system.

(4) Disturbance rejection properties are limited by signal saturation in the control loop.

\section{REFERENCES}

[1] Hu, F. and Edge, K. A., Adaptive Control of a Piston Pump, Proceedings of the Fourth Bath International Fluid Power Workshop on Systems, Modelling, and Control, Research Studies Press, September 1991.

[2] Hori, N., Ukrainetz, p. R., Nikiforuk, P. N. and Bitner, D. V., Robust Discrete-time Adaptive Control of an Electrohydraulic Servo Actuator, Proceedings of 8th International Symposium on Fluid Power, pp. 495-514, 1988.

[3] Vaughan, N. D., and Plummer, A. R. Robust Adaptive Control for Hydraulic Servosystems, ASME Winter Annual Meeting, Dallas, Texas, November 25-30, 1990.

[4] Aström, K. J., Hagander, P., and Sternby, J., Zeros of Sampled Systems, Automatica, Vol. 20, No. 1, pp. 31-38, 1984.

[5] Goodwin, G. C., Leal, R. Lozano, Mayne, D. Q., and Middleton, R. H., Rapprochement between Continuous and Discrete Model Reference Adaptive Control, Automatica, Vol. 22, No. 2, pp. 199-207, 1986.

[6] Dariusz Janecki, Model Reference Adaptive Control Using Delta Operator, IEEE Trans. on Automatic Control, Vol. 33, No. 8, pp.771-774, 1988.

[7] Suzuki, T., and Tanaka, K., Expression of Generalized Adaptive Law Using Delta Operator, Proceeding of IFAC Symposium on Adaptive System Control \& Signal Processing, Vol J, Glasgow, 19-21 April 1989.

[8] Egardt, B., Unification of Some Continuous-time Adaptive Schemes, IEEE Trans. Automatic Control, AC-24, pp. 588-592, 1979. 